\title{
Polycystic ovary syndrom(PCOS) and the association of subsequent risk of Graves' Diseases: a population-based cohort study
}

\author{
Ming-Li Chen ${ }^{1}$, Jia-Ying Lin ${ }^{1}$, Kun-Hong Li ${ }^{2}$, Hei-Tung Yip ${ }^{3}$, and James Cheng-Chung \\ $\mathrm{Wei}^{2}$ \\ ${ }^{1}$ Chung Shan Medical University College of Medicine \\ ${ }^{2}$ Chung Shan Medical University \\ ${ }^{3}$ China Medical University Hospital
}

November 9, 2020

\begin{abstract}
Objective: This study aimed to investigate the risk of Graves' disease in Polycystic ovary syndrome (PCOS) patients. Design: Population-based retrospective cohort study. Setting: We utilized the medical records randomly selected from the Taiwan National Health Insurance Research Database (NHIRD) during period between 2000 and 2013. Population: We included women aged between 20 and 50 who have no previous Graves' disease diagnosis records. Methods: We matched the baseline characteristics and the index date of the PCOS-free cohort to the PCOS cohort at a ratio of 4:1 by propensity score matching method. Cox proportional hazards model was used to estimate hazard ratios (HRs) and 95\% confidence intervals (CIs). Main outcome measure: To analyze the cumulative incidence and the hazard ratio of Graves' disease events in both PCOS group and PCOS-free cohort. Results: We identified 5927 patients as the PCOS cohort, and included 23708 patients as the PCOS-free cohort. The PCOS group had a significant increased risk of Graves' disease with an adjusted hazard ratio (aHR) of 1.34 (95\% CI $=1.00-1.81)$. The p-value was 0.03 in log-rank test. PCOS patients without anxiety, chronic obstructive pulmonary disease, hypertension or hyperlipidemia had a higher risk of Graves' disease in the stratification analysis. Conclusions: This study revealed a significant association between PCOS and subsequent risk of developing Graves' disease. Funding: Nil. Tweetable abstract: PCOS increase the risk of Graves' disease. Keywords: Polycystic ovary syndrome, PCOS, Graves' disease, GD, hyperthyroidism, autoimmune thyroiditis, cohort, hazard ratio, epidemiology, Taiwan National Health Insurance Research Database
\end{abstract}

\section{Introduction}

Polycystic ovary syndrome (PCOS) is recognized as one of the most common endocrine disorders in women, causing ovulatory and menstrual irregularity, infertility, hyperandrogenism, and metabolic dysfunction. The association of PCOS and autoimmune diseases, especially autoimmune thyroiditis, is increasingly being recognized. Graves' disease is an autoimmune thyroiditis affecting mostly young women, and causes symptoms such as hyperthyroidism, sweating, heat intolerance, weight loss, increased appetite, and tremor. $(1,2)$ Characterized by lymphocytic infiltration of the thyroid parenchyma, Graves' disease is found to be regulated by T helper 1 (Th1) lymphocytes, several cytokines, and chemokines.(3) Thyrotropin receptor autoantibodies (TRAb) act as the important indicator of the development of Graves' disease, which is often used in clinical assessment. $(4,5)$ Although the exact pathogenic mechanism is not fully clear to date, an individual's genetic predisposition, interactions between endogenous and environmental factors, and immune system dysregulation greatly contribute to the onset of Graves' disease. $(6,7)$

Previous studies showed that patients with PCOS run an elevated risk of autoimmune thyroiditis compared with those without PCOS. $(8,9)$ A previous clinical trial proved a positive relationship between ovary func- 
tion, thyroid function, and insulin resistance in women.(10) A previous systematic review also revealed a higher prevalence of autoimmune thyroid diseases in patients with PCOS compared with those without.(11) A Danish cohort study further indicated that patients with PCOS had a higher risk to develop thyroid diseases.(12) However, only few pieces of research exclusively explored the association between PCOS and Graves's disease. A previous case series of 6 patients indicated a positive relationship of PCOS and Graves' disease.(13) A higher prevalence of serological indicator of Graves' disease has also been found in PCOS patients.(14) Since the epidemiology of the relationship of PCOS and new-onset Graves' disease hasn't been well disclosed, we conducted this original longitudinal nationwide cohort study to explore this issue.

\section{Methods}

\section{Data source}

The data form the Taiwan National Health Insurance Research Database (NHIRD) was analyzed in this study. The Taiwan National Health Insurance (NHI) program launched in 1995 and covered 99\% of Taiwan residents. The comprehensive claims data form NHI program was collected into NHIRD. We utilized the outpatients and in-patient records of one million beneficiaries, the Longitudinal Health Insurance Database (LHID). The database used the International Classification of Diseases, Ninth Revision, Clinical Modification (ICD-9-CM) codes to define the corresponding diseases. Due to safety and privacy concerns, the identification information was encrypted. This study was approved by the International Review Board (IRB) of China Medical University and Hospital (IRB permit number: CMUH-104-REC2-115).

\section{Study population}

This cohort study was conducted retrospectively from 2000 to 2013. Female with newly diagnosed with polycystic ovary syndrome (PCOS), with at least two outpatient visits or once admission record, were enrolled as PCOS cohort. The inclusion criteria were female aged from 20 to 50 years old, and have never been diagnosed with Graves' disease before. We exclude patients with a history of thyroid diseases or comorbidities that might have caused Graves' disease: congenital hypothyroidism (ICD-9-CM code 243), postsurgical hypothyroidism (ICD-9-CM code 244.0), other postablative hypothyroidism (ICD-9-CM code 244.1), iodine hypothyroidism (ICD-9-CM code 244.2), other iatrogenic hypothyroidism (ICD-9-CM code 244.3), iatrogenic thyroiditis (ICD-9-CM code 245.4), and thyroid dysfunction (ICD-9-CM code 648.1). The PCOS-free female was recruited as the PCOS-free cohort. We matched one PCOS patient to four PCOS-free patients according to age and index year. The index date was designed to be the first date of PCOS diagnosis for PCOS cohort, and a random date between 2000 and 2012 for the PCOS-free cohort. We observed the participant until they started a Graves' disease event, loss of follow-up, or the end of the study, whichever came first.

\section{Main outcome and comorbidities}

Graves' disease was the primary end point in this study. We defined Graves' disease patients as patients with two or more outpatient visit, or one in-patient records of ICD code 242.0. The following comorbidities developed before the index date were considered as confounders: anxiety (ICD-9-CM 309.2-309.4), asthma (ICD-9-CM 493), coronary artery disease (CAD) (ICD-9-CM 410-413, 414.01-414.05, 414.8, and 414.9), cancer (ICD-9-CM 140-208), chronic obstructive pulmonary disease (COPD) (ICD-9-CM 490-496), congestive heart failure (CHF) (ICD-9-CM 398.91, 402.01, 402.11, 402.91, and 428), chronic liver diseases (CLD) (ICD-9-CM 571.4), diabetes mellitus (DM) (ICD-9-CM 250), depression (ICD-9-CM 296.2, 296.3, 296.5, 296.82, 300.4, 309.0-309.1 and 311), hypertension (ICD-9-CM 401-405), hyperlipidemia (ICD-9-CM 272), stroke (ICD-9-CM 430-438) and sleep apnea (ICD-9-CM 327.2, 780.51, 780.53, 780.57).

\section{Statistical analysis}

The variables were presented as percentages for categorical variables, and as mean for continuous variables. We examined the baseline variables of the PCOS cohort and non-PCOS cohort by chi-square tests and student's t-tests. The Kaplan-Meier method was used to acquire the cumulative incidence curve for the two cohorts. Log-rank test was also performed. The univariate Cox proportional hazard (PH) model was 
applied to estimate the hazard ratio and then adjusted the hazard ratio by multivariate Cox PH model. All statistical analyses were conducted with SAS 9.4 software (SAS Institute, Cary, NC, USA). P-value of less than 0.05 was regarded as significant.

\section{Results}

As shown in Table 1, we included 5927 patients with PCOS from 2000 to 2012 and 23708 patients without PCOS. The mean follow-up time of the case cohort was $6.80( \pm 3.56)$ years. The participants in our study were mainly aged from $20-30$ years old $(60 \%)$. The mean age was about 29 years old. The proportion of patients with comorbidities was similar in both cohorts.

The incidence rate of Graves' diseases for PCOS patients was 1.46 per 1000 person-years and that of nonPCOS patients was 1.06 per 1000 person-years (Table 2.). Figure 1 presented the cumulative incidence curve of Graves' diseases in patients with PCOS was significantly higher than that in the patient without PCOS (Log-rank test: p-value=0.03). The adjusted hazard ratio (aHR) of Graves' disease for the PCOS cohort relative to the non-PCOS cohort was $1.34(95 \% \mathrm{CI}=1.00-1.81)$. Patients with CAD and DM increased the risk of Graves' disease by $2.66(95 \% \mathrm{CI}=1.08-6.56)$ and $2.17(95 \% \mathrm{CI}=1.06,4.44)$ folds, respectively.

Table 3 presents the stratification analysis of the association of PCOS and Graves' disease in each age group and comorbidities. PCOS patients aged 31-40 had a higher risk of Graves' disease (aHR=2.31, 95\% CI =1.483.61). The independent association of PCOS with Graves' disease was significant in patients without anxiety, COPD, or hypertension. For patients with hyperlipidemia, PCOS raises the risk of Graves' disease by 10.2 $(95 \% \mathrm{CI}=1.21,85.1)$ times.

\section{Structured Discussion}

\section{Principal Findings}

This 13-year retrospective cohort study revealed an increased incidence of Graves' diseases in PCOS patients with an aHR of 1.34 (95\%C.I. 1.00-1.81) after adjusting for age and confounders listed in table 1. In stratification analysis, the independent association of PCOS with Graves' disease was especially significant in group aged 31 to $40(\mathrm{aHR}=2.31,95 \%$ C.I. 1.48-3.61). The cluster of PCOS and Graves' disease at this age may due to the nature of PCOS, a common endocrinopathy of reproductive age-group women. Several comorbidities were found influential in our Cox regression model, such as CAD ( $\mathrm{aHR}=2.66,95 \%$ C.I. 1.08-6.56) and $\mathrm{DM}(\mathrm{aHR}=2.17,95 \%$ C.I. 1.06-4.44). In our stratification study investigating into the association of PCOS and Graves' diseases in different subgroups, we found that in patients with hypertension, the independent association of PCOS with Graves' disease became insignificant, which may be explainable due to probable effect of other coexisting comorbidities and relatively small numbers of events. However, in patients with hyperlipidemia, PCOS increases the risk of Graves' diseases by 10 folds, higher than PCOS acting alone. In general, after adjusting for confounders in our study, the overall independent association of PCOS with Graves' disease remains strong, which may supports the hypothesis of PCOS as a possible risk factor to the development of Graves' diseases.

Results

Previous studies highlight the association of Graves' diseases and PCOS was presented, although comparably rare to hypothyroidism events. $(12,13,15,16)$ In previous case series, the clinical observation of coexisting of definite PCOS and active form of Graves' disease emphasized the role of autoimmunity in the development of PCOS, suggesting a common pathogenic linkage between these two diseases.(13) The risk of Graves' disease in PCOS was reported more than two times higher than controls in a previous epidemiology study.(12) Higher prevalence of TRAb, which is crucial in the evaluation of Graves' disease,(17) has also been described in PCOS patients.(14)

\section{Research Implications}

One theoretical mechanism is hyperestrogenic state in PCOS. Hyperestrogenism increased autoimmune diseases by having a proliferative action on B-lymphocytes, T-cell and macrophages.(18, 19) The positive 
result of our study serves as a retrospective population-based evidence to sustain previous observations of the interplay between the two clinical conditions. The relationship between PCOS and Graves' diseases might be more than a mere coincident finding in coexistence.

\section{Clinical Implications}

Metabolic comorbidities are also note-worthy in interpreting the risk of Graves' diseases in PCOS. In our study, patients with DM and CAD have more risk to develop Graves' diseases. Adverse metabolic outcomes in PCOS have been widely discussed in previous studies, such as impaired glucose tolerance, insulin resistance, DM, cardiovascular disease, and metabolic syndrome.(20) Obesity exacerbates many of these subsequent outcomes such as DM.(21) Higher metabolic inflexibility was observed to be associated with hyperandrogenemia and insulin resistance in PCOS patients,(22) as a result of an inflammation status triggered by saturated fat ingestion, may be a separate and distinct contributor independent from obesity.(23) Obesity increases leptin and TRH level, which further raise TSH and induce proliferation of adipocytes again via TSH receptors. Leptin also lead to autoimmunity by up-regulating effector T-cells and down-regulating regulator T-cells.(24)

Additionally, in our study, patients with depression were prone to develop Graves' diseases with a subtle but insignificant aHR of 1.57 (95\%C.I. 0.92-2.67). Little but consistent evidence for psychological issues in PCOS patients was presented in previous studies.(25-28) Objective measurements such as obesity, insulin resistance, and elevated serum androgens might partly contribute to the association of depression and PCOS, but the conclusion remains controversial. $(27,28)$ Since comorbidities sustained in our study were proven to reduce the quality of life for patients with PCOS, physicians are suggested to closely monitor the progression of high-risk comorbidities in PCOS patients.

\section{Strengths and Limitations}

The main strength of the study was the sizable long-term population-based patient group included in our study. The advantages of using the NHIRD such as long-term comprehensive follow-up and universal coverage has been described in a previous study.(29) We excluded patients with previous thyroid diseases due to possible interplay of a history of thyroid diseases that might have caused Graves' diseases. We also qualified the definition of PCOS events in our study to more than twice outpatient or one hospitalized record. Generally, our database size ensures similar distributions due to well-balanced matching, and reduces the heterogeneity and selection bias.

There are some limitations to our study. Since PCOS and autoimmune thyroid diseases, including Graves' diseases, have a familial clustering phenomenon, an alternative explanation may be suggested to a common genetic pathway.(30) However, the genetic background of individuals was not available in our NHIRD scheme. The development of Graves' diseases may depend on different ethnic groups. Lab data such as hormone level was also not available in NHIRD, posing limitations in further addressing the association of the role of hormones in the association of PCOS and Graves' diseases. Although the Bureau of NHI uses strict auditing mechanisms to reimburse insurance claims, the ICD-9 codes claimed from the NHIRD might be inaccurate due to diagnostic uncertainty and misclassification in reality. The symptoms of PCOS and Graves' diseases may sometimes be too mild to be recognized by patients. Thus, the underrepresentation of the identified population may occur and eclipse the accuracy of prevalence.

\section{Conclusion}

Our study revealed a positive finding of the association between PCOS and Graves' diseases. Beside from known importance of screening for common comorbidities in PCOS patients, our findings stress the need for monitoring for thyroid diseases during follow-up.

\section{Ethics statement}

This study was approved by the International Review Board (IRB) of China Medical University and Hospital (IRB permit number: CMUH-104-REC2-115). The need of informed consent was waived. 


\section{Declaration of interest}

The authors report no conflict of interest.

\section{Contribution to authorship}

Ming-Li Chen: Conceptualization, Writing-Original draft preparation, Writing-Review and editing JiaYing Lin:Writing-Original draft preparation Kun-Hong Li: Writing-Review and editing, Supervision Hei-Tung Yip: Methodology, Software, Investigation, Data curation, Writing-Original draft preparation, Visualization James Cheng-Chung Wei: Writing-Review and editing, Supervision, Project administration

\section{Funding}

There is no funding in this research.

\section{Acknowledgment}

This study is supported in part by Taiwan Ministry of Health and Welfare Clinical Trial Center (MOHW109TDU-B-212-114004), MOST Clinical Trial Consortium for Stroke (MOST 108-2321-B-039-003-), Tseng-Lien Lin Foundation, Taichung, Taiwan.

\section{Reference}

1. Antonelli A, Ferrari SM, Ragusa F, Elia G, Paparo SR, Ruffilli I, et al. Graves' disease: Epidemiology, genetic and environmental risk factors and viruses. Best Pract Res Clin Endocrinol Metab. 2020:101387. Epub 2020/02/29. doi: 10.1016/j.beem.2020.101387. PubMed PMID: 32107168.

2. Wemeau JL, Klein M, Sadoul JL, Briet C, Velayoudom-Cephise FL. Graves' disease: Introduction, epidemiology, endogenous and environmental pathogenic factors. Ann Endocrinol (Paris). 2018;79(6):599607. Epub 2018/10/22. doi: 10.1016/j.ando.2018.09.002. PubMed PMID: 30342794.

3. Antonelli A, Ferrari SM, Corrado A, Di Domenicantonio A, Fallahi P. Autoimmune thyroid disorders. Autoimmun Rev. 2015;14(2):174-80. Epub 2014/12/03. doi: 10.1016/j.autrev.2014.10.016. PubMed PMID: 25461470.

4. Ross DS, Burch HB, Cooper DS, Greenlee MC, Laurberg P, Maia AL, et al. 2016 American Thyroid Association Guidelines for Diagnosis and Management of Hyperthyroidism and Other Causes of Thyrotoxicosis. Thyroid. 2016;26(10):1343-421. Epub 2016/08/16. doi: 10.1089/thy.2016.0229. PubMed PMID: 27521067.

5. Davies TF, Ando T, Lin RY, Tomer Y, Latif R. Thyrotropin receptor-associated diseases: from adenomata to Graves disease. J Clin Invest. 2005;115(8):1972-83. Epub 2005/08/03. doi: 10.1172/jci26031. PubMed PMID: 16075037; PubMed Central PMCID: PMCPMC1180562.

6. Ajjan RA, Weetman AP. The Pathogenesis of Hashimoto's Thyroiditis: Further Developments in our Understanding. Horm Metab Res. 2015;47(10):702-10. Epub 2015/09/12. doi: 10.1055/s-0035-1548832. PubMed PMID: 26361257.

7. Menconi F, Marcocci C, Marino M. Diagnosis and classification of Graves' disease. Autoimmun Rev. 2014;13(4-5):398-402. Epub 2014/01/16. doi: 10.1016/j.autrev.2014.01.013. PubMed PMID: 24424182.

8. Kim JJ, Yoon JW, Kim MJ, Kim SM, Hwang KR, Choi YM. Thyroid autoimmunity markers in women with polycystic ovary syndrome and controls. Hum Fertil (Camb). 2020:1-7. Epub 2020/01/08. doi: 10.1080/14647273.2019.1709668. PubMed PMID: 31910041.

9. Ulrich J, Goerges J, Keck C, Muller-Wieland D, Diederich S, Janssen OE. Impact of Autoimmune Thyroiditis on Reproductive and Metabolic Parameters in Patients with Polycystic Ovary Syndrome. Exp Clin Endocrinol Diabetes. 2018;126(4):198-204. Epub 2018/03/06. doi: 10.1055/s-0043-110480. PubMed PMID: 29506313. 
10. Pallotti S, Gasbarrone A, Franzese IT. [Relationship between insulin secretion, and thyroid and ovary function in patients suffering from polycystic ovary]. Minerva Endocrinol. 2005;30(3):193-7. Epub 2005/10/07. PubMed PMID: 16208308.

11. Romitti M, Fabris VC, Ziegelmann PK, Maia AL, Spritzer PM. Association between PCOS and autoimmune thyroid disease: a systematic review and meta-analysis. Endocr Connect. 2018;7(11):1158-67. Epub 2018/10/24. doi: 10.1530/ec-18-0309. PubMed PMID: 30352422; PubMed Central PMCID: PMCPMC6215798.

12. Glintborg D, Rubin KH, Nybo M, Abrahamsen B, Andersen M. Increased risk of thyroid disease in Danish women with polycystic ovary syndrome: a cohort study. Endocr Connect. 2019;8(10):1405-15. Epub 2019/09/14. doi: 10.1530/ec-19-0377. PubMed PMID: 31518989; PubMed Central PMCID: PMCPMC6826171.

13. Nisar S, Shah PA, Kuchay MS, Bhat MA, Rashid A, Ahmed S, et al. Association of polycystic ovary syndrome and Graves' disease: Is autoimmunity the link between the two diseases. Indian J Endocrinol Metab. 2012;16(6):982-6. Epub 2012/12/12. doi: 10.4103/2230-8210.103006. PubMed PMID: 23226647; PubMed Central PMCID: PMCPMC3510972.

14. Mobeen H, Afzal N, Kashif M. Polycystic Ovary Syndrome May Be an Autoimmune Disorder. Scientifica (Cairo). 2016;2016:4071735. Epub 2016/06/09. doi: 10.1155/2016/4071735. PubMed PMID: 27274883; PubMed Central PMCID: PMCPMC4871972.

15. Jung JH, Hahm JR, Jung TS, Kim HJ, Kim HS, Kim S, et al. A 27-year-old woman diagnosed as polycystic ovary syndrome associated with Graves' disease. Intern Med. 2011;50(19):2185-9. Epub 2011/10/04. doi: 10.2169/internalmedicine.50.5475. PubMed PMID: 21963738.

16. Trolle B, Flyvbjerg A, Kesmodel U, Lauszus FF. Efficacy of metformin in obese and non-obese women with polycystic ovary syndrome: a randomized, double-blinded, placebo-controlled cross-over trial. Hum Reprod. 2007;22(11):2967-73. Epub 2007/09/04. doi: 10.1093/humrep/dem271. PubMed PMID: 17766923.

17. Kotwal A, Stan M. Thyrotropin Receptor Antibodies-An Overview. Ophthalmic Plast Reconstr Surg. 2018;34(4S Suppl 1):S20-s7. Epub 2018/05/18. doi: 10.1097/iop.0000000000001052. PubMed PMID: 29771756 .

18. Fairweather D, Rose NR. Women and autoimmune diseases. Emerg Infect Dis. 2004;10(11):2005-11. Epub 2004/11/20. doi: 10.3201/eid1011.040367. PubMed PMID: 15550215; PubMed Central PMCID: PMCPMC3328995.

19. Cutolo M, Sulli A, Straub RH. Estrogen metabolism and autoimmunity. Autoimmun Rev. 2012;11(67):A460-4. Epub 2011/12/14. doi: 10.1016/j.autrev.2011.11.014. PubMed PMID: 22155198.

20. Gilbert EW, Tay CT, Hiam DS, Teede HJ, Moran LJ. Comorbidities and complications of polycystic ovary syndrome: An overview of systematic reviews. Clin Endocrinol (Oxf). 2018;89(6):683-99. Epub 2018/08/14. doi: 10.1111/cen.13828. PubMed PMID: 30099747.

21. Forslund M, Landin-Wilhelmsen K, Trimpou P, Schmidt J, Brannstrom M, Dahlgren E. Type 2 diabetes mellitus in women with polycystic ovary syndrome during a 24-year period: importance of obesity and abdominal fat distribution. Hum Reprod Open. 2020;2020(1):hoz042. Epub 2020/01/25. doi: 10.1093/hropen/hoz042. PubMed PMID: 31976382; PubMed Central PMCID: PMCPMC6964225.

22. Rimmer M, Tan BK, Teede H, Thangaratinam S, B HAW. Metabolic inflexibility in women with polycystic ovary syndrome: a systematic review. Gynecol Endocrinol. 2019:1-7. Epub 2019/12/04. doi: 10.1080/09513590.2019.1698025. PubMed PMID: 31793357.

23. Gonzalez F, Considine RV, Abdelhadi OA, Acton AJ. Inflammation triggered by saturated fat ingestion is linked to insulin resistance and hyperandrogenism in PCOS. J Clin Endocrinol Metab. 2020. Epub 2020/03/07. doi: 10.1210/clinem/dgaa108. PubMed PMID: 32140727. 
24. Matarese G, Leiter EH, La Cava A. Leptin in autoimmunity: many questions, some answers. Tissue Antigens. 2007;70(2):87-95. Epub 2007/07/06. doi: 10.1111/j.1399-0039.2007.00886.x. PubMed PMID: 17610413.

25. Chen SF, Yang YC, Hsu CY, Shen YC. Risk of schizophrenia in patients with polycystic ovary syndrome: a nationwide population-based cohort study from Taiwan. J Psychosom Obstet Gynaecol. 2020:1-7. Epub 2020/03/07. doi: 10.1080/0167482x.2020.1735342. PubMed PMID: 32141403.

26. Chen SF, Yang YC, Hsu CY, Shen YC. Risk of bipolar disorder in patients with polycystic ovary syndrome: A nationwide population-based cohort study. J Affect Disord. 2020;263:458-62. Epub 2020/01/24. doi: 10.1016/j.jad.2019.12.007. PubMed PMID: 31969278.

27. Cooney LG, Dokras A. Depression and Anxiety in Polycystic Ovary Syndrome: Etiology and Treatment. Curr Psychiatry Rep. 2017;19(11):83. Epub 2017/09/21. doi: 10.1007/s11920-017-0834-2. PubMed PMID: 28929349

28. Greenwood EA, Pasch LA, Cedars MI, Legro RS, Huddleston HG. Association among depression, symptom experience, and quality of life in polycystic ovary syndrome. Am J Obstet Gynecol. 2018;219(3):279.e1.e7. Epub 2018/07/04. doi: 10.1016/j.ajog.2018.06.017. PubMed PMID: 29969586; PubMed Central PMCID: PMCPMC6758922.

29. Hsing AW, Ioannidis JP. Nationwide Population Science: Lessons From the Taiwan National Health Insurance Research Database. JAMA Intern Med. 2015;175(9):1527-9. Epub 2015/07/21. doi: 10.1001/jamainternmed.2015.3540. PubMed PMID: 26192815.

30. Li Q, Yang G, Wang Y, Zhang X, Sang Q, Wang H, et al. Common genetic variation in the 3'-untranslated region of gonadotropin-releasing hormone receptor regulates gene expression in cella and is associated with thyroid function, insulin secretion as well as insulin sensitivity in polycystic ovary syndrome patients. Hum Genet. 2011;129(5):553-61. Epub 2011/01/29. doi: 10.1007/s00439-011-0954-4. PubMed PMID: 21274726.

Figure 1. The cumulative incidence of Graves's diseases in patients with and without PCOS.

Table 1. Baseline characteristics of non-PCOS cohort and PCOS cohort.

\footnotetext{
Variables

Age, year

20-30

31-40

41-50

mean, (SD)

Comorbidities

asthma

anxiety

CAD

cancer

COPD

$\mathrm{CHF}$

CLD

diabetes

depression

hypertension

hyperlipidemia

stroke
} 
sleep apnea

CAD: coronary artery disease; COPD: chronic obstructive pulmonary disease; CHF: congestive heart failure; CLD: chronic

Table 2. Incidence rate and hazard ratio of Graves's diseases.

Variables

All

non-PCOS

PCOS

Age, year

14-20

21-50

$>50$

Comorbidities

asthma

No

Yes

anxiety

No

Yes

CAD

No

Yes

cancer

No

Yes

COPD

No

Yes

CHF

No

Yes

CLD

No

Yes

diabetes

No

Yes

depression

No

Yes

hypertension

No

Yes

hyperlipidemia

No

Yes

stroke 
No

Yes

sleep apnea

No

Yes

: p-value $<0.05 ; * *:$ p-value $<0.01 ; * * *$ : p-value $<0.001 ;$ PY: person-years; IR: incidence rate (per 1000 person-years); cHR:

Table 3. Incidence rate and hazard ratio of Graves's diseases stratified by age and comorbidities.

Variables

Age, year

20-30

$31-40$

41-50

Comorbidities

asthma

No

Yes

anxiety

No

Yes

CAD

No

Yes

cancer

No

Yes

COPD

No

Yes

CHF

No

Yes

CLD

No

Yes

diabetes

No

Yes

depression

No

Yes

hypertension

No

Yes

hyperlipidemia

No

Yes 
stroke

No

Yes

sleep apnea

No

Yes

: p-value $<0.05 ;{ }^{* *}:$ p-value $<0.01 ; * * *$ p-value $<0.001 ;$ PY: person-years; IR: incidence rate (per 1000 person-years); cHR: c

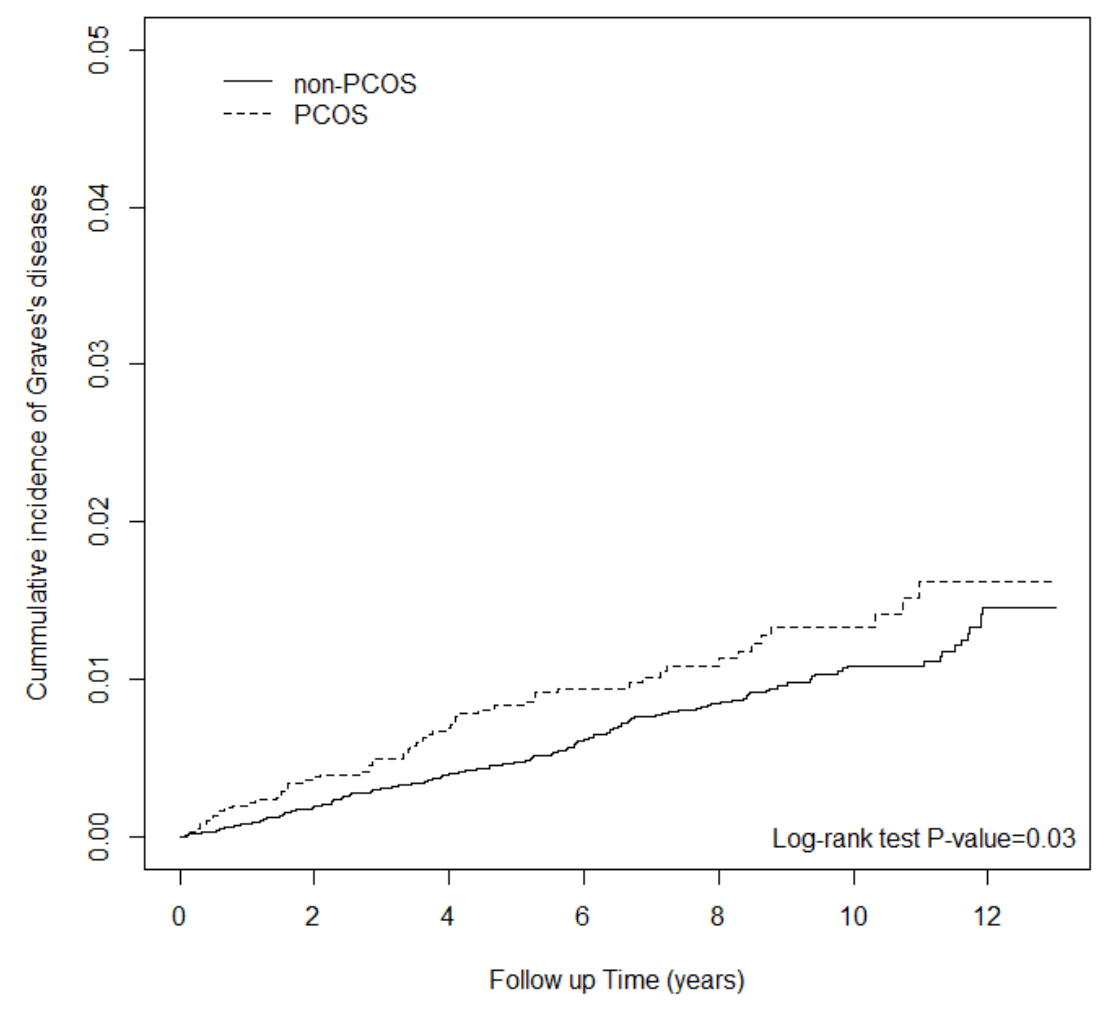

